\title{
THE GROUP OF ORDER PRESERVING AUTOMORPHISMS OF AN ORDERED ABELIAN GROUP
}

\author{
PAUL CONRAD
}

In this note we shall use the terminology and notation from [2, pp. 516-517]. In particular, order will always mean linear order. Some answers are given to the question: When can the group $\mathfrak{A}$ of $o$-automorphisms of an abelian $o$-group $G$ be ordered? We prove that $\mathfrak{A}$ can be ordered provided that the rank of $G$ is well ordered and the $o$-automorphism group of each component of $G$ is isomorphic to a subgroup of the positive rationals. Thus every torsion free abelian group $G$ admits an ordering for which the corresponding $\mathfrak{A}$ can be ordered. Our results provide examples of nonabelian 0 -groups, and they can be used to obtain information about the underlying group $G$.

1. Representation of an automorphism group by a group of matrices. Whenever possible we shall represent automorphisms by matrices. This is a straightforward generalization of the classical case (for example, see Kurosh [3, p. 156]), and no proofs will be given. Let $\Gamma$ be an ordered set, and for each $\gamma \in \Gamma$, let $D_{\gamma}$ be an abelian group. We use the same symbol 0 to denote the identity element of each of the $D_{\gamma}$. Let $E_{\gamma}$ be the ring of endomorphisms of $D_{\gamma}$, and let $H\left(D_{\alpha}, D_{\beta}\right)$, where $\alpha, \beta \in \Gamma$ and $\alpha \neq \beta$, be the group of homomorphisms of $D_{\alpha}$ into $D_{\beta}$. Finally let $G$ be the restricted direct sum of the $D_{\gamma}$, and let $\Delta$ be the set of all square matrices $\left[\pi_{\alpha \beta}\right]$, where $\alpha, \beta \in \Gamma$, $\pi_{\alpha \alpha} \in E_{\alpha}$, and $\pi_{\alpha \beta} \in H\left(D_{\alpha}, D_{\beta}\right)$ for $\alpha \neq \beta$, that satisfy:

$\left.{ }^{*}\right)$ For $\alpha$ fixed and each $d \in D_{\alpha}, d \pi_{\alpha \beta} \neq 0$ for at most a finite number of the $\beta$.

$$
\begin{aligned}
& \text { If }\left(\cdots, d_{\alpha}, \cdots\right) \in G \text { and }\left[\pi_{\alpha \beta}\right] \in \Delta \text {, then } \\
& \quad\left(\cdots, d_{\alpha}, \cdots\right)\left[\pi_{\alpha \beta}\right]=\left(\cdots, \sum_{\alpha \in \Gamma} d_{\alpha} \pi_{\alpha \beta}, \cdots\right) \in G .
\end{aligned}
$$

It is easy to verify that this mapping is an endomorphism of $G$. Conversely if $\pi$ is an endomorphism of $G$, then $\left(0, \cdots, 0, a_{\alpha}, 0, \cdots, 0\right) \pi$ $=\left(\cdots, b_{\beta}, \cdots\right)$ where at most a finite number of the $b_{\beta}$ are nonzero. Define $a_{\alpha} \pi_{\alpha \beta}=b_{\beta}$, then we have a mapping of $\pi$ upon $\left[\pi_{\alpha \beta}\right] \in \Delta$. This mapping is an isomorphism of the ring of all endomorphisms of $G$ onto $\Delta$. Suppose that each $D_{\gamma}$ is an o-group, and define $\left(\cdots, d_{\gamma}, \cdots\right) \in G$ positive if it is not zero and the nonzero com-

Received by the editors November 9, 1957.

1 This research was supported by a grant from the National Science Foundation. 
ponent $d_{\gamma}$ with greatest subscript $\gamma$ is positive. Then $G$ is an abelian $o$-group, and for each $\gamma \in \Gamma, C_{\gamma}=\left\{\left(\cdots, g_{\alpha}, \cdots\right) \in G: g_{\alpha}=0\right.$ for all $\alpha>\gamma\}$ is a convex subgroup of $G$. Let $\mathfrak{A}$ be the group of all $o$-automorphisms of $G$, and for each $\gamma \in \Gamma$, let $\mathfrak{D}_{\gamma}$ be the group of all $o$ automorphisms of $D_{\gamma}$. Let $T=T\left(\Gamma, D_{\gamma}\right)$ be the multiplicative semigroup of all the triangular matrices $\left[\pi_{\alpha \beta}\right] \in \Delta$ that satisfy:

(a) $\pi_{\gamma \gamma} \in \mathfrak{D}_{\gamma}$ for all $\gamma \in \Gamma$.

(b) If $\alpha, \beta \in \Gamma, \beta>\alpha$ and $d \in D_{\alpha}$, then $d \pi_{\alpha \beta}=0$.

$\left[\pi_{\alpha \beta}\right] \in T$ corresponds to an $o$-isomorphism of $G$ into itself, and $\left[\pi_{\alpha \beta}\right]$ corresponds to an $o$-automorphism of $G$ if and only if $\left[\pi_{\alpha \beta}\right]$ is a unit in $T$. Let $U=U\left(\Gamma, D_{\gamma}\right)$ be the group of units of $T$.

LEMma 1. If each of the convex subgroups $C_{\gamma}$ of $G$ is invariant with respect to $\mathfrak{A}$, then $\mathfrak{A}$ is isomorphic to $U$.

Proof. By the isomorphism defined in the preceding discussion, $\mathfrak{A}$ is mapped onto a subgroup of the group of all matrices in $\Delta$ that have inverses in $\Delta$. Let $\pi \in \mathfrak{A}$ and let $\left[\pi_{\alpha \beta}\right]$ be the corresponding matrix. For each $\gamma \in \Gamma, C_{\gamma}\left[\pi_{\alpha \beta}\right]=C_{\gamma}$. It follows that $\left[\pi_{\alpha \beta}\right]$ satisfies (b). Then since $\pi$ is an $o$-automorphism, $\left[\pi_{\alpha \beta}\right]$ satisfies (a).

CoRollaRy. If each $D_{\gamma}$ is a subgroup of the reals (naturally ordered), and the only o-permutation of $\Gamma$ is the identity permutation, then $\mathfrak{A}$ is isomorphic to $U$.

For under these restrictions it is easy to verify that every convex subgroup of $G$ is invariant with respect to $\mathfrak{A}$.

REMARK. If each $D_{\gamma}$ is the group of reals, then $\mathfrak{A}$ is isomorphic to $U$ if and only if the only $o$-permutation of $\Gamma$ is the identity permutation.

A subgroup $S$ of $G$ is a c-subgroup if for every $\gamma \in \Gamma$ and $d \in D_{\gamma}$ there exists an element $\left(\cdots, g_{\alpha}, \cdots\right) \in S$ such that $g_{\gamma}=d$ and $g_{\alpha}=0$ for all $\alpha>\gamma$.

Lemma 2. Suppose that each $D_{\gamma}$ is a nonzero d-closed subgroup of the reals. Then $U=T$ if and only if $\Gamma$ is well ordered.

Proof. Each $\left[\pi_{\alpha \beta}\right] \in T$ induces an $o$-isomorphism of $G$ onto a $c$ subgroup of $G$. If $\Gamma$ is well ordered, then $G$ has no proper $c$-subgroups $\left[1\right.$, p. 8]. Thus $G\left[\pi_{\alpha \beta}\right]=G$ for all $\left[\pi_{\alpha \beta}\right] \in T$, hence $U=T$.

Conversely assume that $\Gamma$ is not well ordered. Then we can find a set $\left\{h_{\gamma_{i}}\right\}_{i=1}^{\infty}$ of nonzero elements such that $h_{\gamma_{i}} \in D_{\gamma_{i}}$ and $\gamma_{1}>\gamma_{2}>\gamma_{3}>\ldots$. Let $h_{\gamma_{i}}^{*}$ be the smallest $d$-closed subgroup of $D_{\gamma_{i}}$ that contains $h_{\gamma_{i}}$, and let $B$ be the restricted direct sum of the $h_{\gamma_{i}}^{*}$. Define $h_{\gamma_{i}} \pi=h_{\gamma_{i+1}}+h_{\gamma_{i}}$. Since $B$ is a vector space over the rationals 
and the $h_{\gamma_{i}}$ form a basis for $B$, there is a unique extension of $\pi$ to an $o$-isomorphism $\sigma$ of $B$ into $B$. Note that $\left(0, \cdots, 0, h_{\gamma_{1}}, 0, \cdots, 0\right)$ does not belong to $B \sigma$. For each $\gamma_{i}$ let $K_{\gamma_{i}}$ be a subgroup of $D_{\gamma_{i}}$ such that $D_{\gamma_{i}}=h_{\gamma_{i}}^{*} \oplus K_{\gamma_{i}}$. This is possible since the $h_{\gamma_{i}}^{*}$ are $d$-closed. Let $E$ be the restricted direct sum of the $K_{\gamma_{i}}$ for $i=1,2, \cdots$ and the $D_{\alpha}$ for all $\alpha \neq \gamma_{1}, \gamma_{2}, \cdots$. Then $G=B \oplus E$. For each $g=b+e$ in $G$ let $g r$ $=b \sigma+e$. It is easy to verify that $\tau$ is an $o$-isomorphism of $G$ onto a proper subgroup of $G$, and that the corresponding matrix $\left[\pi_{\alpha \beta}\right]$ belongs to $^{2} T$. Thus $U \neq T$.

Corollary. If each $D_{\gamma}$ is a subgroup of the reals and $\Gamma$ is well ordered, then $\mathfrak{A}$ is isomorphic to $U$ and $U=T$.

2. The group $\mathfrak{B}$. For the rest of this paper $G$ will denote an abelian $o$-group, and $\Gamma$ will denote the set of all pairs of convex subgroups $G^{\gamma}, G_{\gamma}$ of $G$ such that $G^{\gamma}$ covers $G_{\gamma}$.

Lemma 3. If $G^{*}$ is the d-closure of $G$ and $\pi$ is an o-automorphism of $G$, then there exists a unique o-automorphism $\pi^{*}$ of $G^{*}$ such that $g \pi^{*}=g \pi$ for all $g \in G$. Moreover the mapping $\pi \rightarrow \pi^{*}$ is an isomorphism of the group $\mathfrak{A}$ of all o-automorphisms of $G$ into the group $\mathfrak{2} *$ of o-automorphisms of $G^{*}$. Thus $\mathfrak{A}$ can be ordered if $\mathfrak{H}^{*}$ can be ordered.

Proof. For each $g^{*} \in G^{*}$ there exists a positive integer $n$ such that $n g^{*} \in G$. Let $g^{*} \pi^{*}$ be the solution of $n x=\left(n g^{*}\right) \pi$. The verification that $\pi^{*} \in \mathfrak{A}^{*}$ and that the mapping $\pi \rightarrow \pi^{*}$ is an isomorphism is not diffcult.

For the remainder of this section we shall assume that $G$ is $d$-closed. Let $\mathfrak{B}=\left\{\pi \in \mathfrak{R}:\left(G_{\gamma}+g\right) \pi=G_{\gamma}+g\right.$ for all $\gamma \in \Gamma$ and all $\left.g \in G^{\gamma}\right\}$. That is, $\mathfrak{B}$ consists of all those $o$-automorphisms of $G$ that induce the identity automorpism on each of the components $G^{\gamma} / G_{\gamma}$ of $G . \mathfrak{B}$ is a normal subgroup of $\mathfrak{A}$. For let $\alpha \in \mathfrak{A}, \beta \in \mathfrak{B}$ and $a \in G^{\gamma}$. $G^{\gamma} \alpha$ and $G_{\gamma} \alpha$ are convex subgroups and $G^{\gamma} \alpha$ covers $G_{\gamma} \alpha$. Therefore $\left(G_{\gamma}+a\right) \alpha \beta \alpha^{-1}$ $=\left(G_{\gamma} \alpha+a \alpha\right) \beta \alpha^{-1}=\left(G_{\gamma} \alpha+a \alpha\right) \alpha^{-1}=G_{\gamma}+a$. Since $G$ is $d$-closed, $G^{\gamma}$ and $G_{\gamma}$ are $d$-closed for all $\gamma \in \Gamma$. Thus $G^{\gamma}=G_{\gamma} \oplus D_{\gamma}$, where $D_{\gamma}$ is a $d$-closed subgroup of $G$. Therefore $D_{\gamma}$ is $o$-isomorphic to a subgroup of the reals. Let $\mathfrak{B}^{\gamma}\left(\mathfrak{B}_{\gamma}\right)$ denote the group of all $o$-automorphisms of $G^{\gamma}\left(G_{\gamma}\right)$ that induce the identity automorphism on all components. By Lemma $1, \mathfrak{B}^{\gamma}$ is isomorphic to the multiplicative group of all matrices of the form

$$
[a]=\left[\begin{array}{ll}
a_{11} & \theta \\
a_{21} & 1
\end{array}\right]
$$

2 The author originally asserted that this was true for an arbitrary choice of the complementary direct summand of $B$, but the referee killed this with a nice counter example. 
where $a_{11} \in \mathfrak{B}_{\gamma}$ and $a_{21} \in H\left(D_{\gamma}, G_{\gamma}\right)$. Here and for the rest of this note 1 will denote an identity automorphism and $\theta$ a zero homomorphism. It will be clear what group 1 refers to ( $D_{\gamma}$ in this case), and what group $\theta$ refers to $\left(H\left(G_{\gamma}, D_{\gamma}\right)\right.$ in this case). In order to show that an ordering of $\mathfrak{B}_{\gamma}$ can be extended to an ordering of $\mathfrak{B}^{\gamma}$ we need the following lemma.

Lemma 4. If $A$ is an abelian o-group, then $H(G, A)$ can be ordered so that if $h \in H$ is positive and $\alpha$ is an o-automorphism of $A$, then ha is positive.

Proof. Since $G$ is torsion free and $d$-closed, it is a vector space over the rationals $\Re$. Choose and well order a basis $g_{1}, g_{2}, \cdots$ for $G$. Define $h \in H$ positive if $h \neq \theta$ and $g_{i} h>0$ in $A$, where $g_{i}$ is the first basis element for which $g_{i} h \neq 0$.

COROLlaRY. $\mathfrak{B}_{\gamma}$ is isomorphic to a subgroup of $\mathfrak{B}^{\gamma}$ and any ordering of $\mathfrak{B}_{\gamma}$ can be extended to an ordering of $\mathfrak{B}^{\gamma}$.

Proof. Order $H\left(D_{\gamma}, G_{\gamma}\right)$ so that if $h \in H$ is positive and $\pi \in \mathfrak{B}_{\gamma}{ }^{\prime}$ then $h \pi$ is positive. Define $[a]$ positive if $a_{11}>1$ or $a_{11}=1$ and $a_{21}>\theta^{\circ}$ It is easy to verify that this definition orders $\mathfrak{B}^{\gamma}$.

Theorem 1. If $\Gamma$ is well ordered, then $\mathfrak{B}$ can be ordered.

Proof. $G$ is isomorphic to the restricted direct sum of the $d$-closed subgroups $D_{\gamma}$ of the reals, where $D_{\gamma} \cong G^{\gamma} / G_{\gamma}[1$, pp. 19 and 8]. Thus by the corollary to Lemma $2, \mathfrak{A}$ is isomorphic to the group of matrices $T\left(\Gamma, D_{\gamma}\right)$. Clearly $\left[\pi_{\alpha \beta}\right] \in T$ corresponds to an element of $\mathfrak{B}$ if and only if $\pi_{\gamma \gamma}=1$ for all $\gamma \in \Gamma . \quad 0=G_{1} \subset G^{1}=G_{2} \subset G^{2}=\cdots \subset G_{\omega} \subset G^{\omega}$ $=\cdots$. Thus for each $\gamma \in \Gamma, \mathfrak{B}^{\gamma}$ is isomorphic to the group of all matrices of the form

$$
\left[\begin{array}{llllllll}
1 & & & & & & \\
a_{21} & 1 & & & \theta & & \\
\vdots & & & & & & \\
a_{\gamma 1} & \cdots & 1 & & & \\
& & & & & & \cdot & \\
& & & & & \cdot & \\
& & & & & 1
\end{array}\right]
$$

Therefore without loss of generality $1=\mathfrak{B}_{1} \subset \mathfrak{B}^{1}=\mathfrak{B}_{2} \subset \mathfrak{B}^{2}=\ldots$ $\subset \mathfrak{B}_{\omega} \subset \mathfrak{B}^{\omega}=\cdots$. If $\gamma \in \Gamma$ has no immediate predecessor, then $\mathfrak{B}_{\gamma}=\bigcup_{\alpha<\gamma} \mathfrak{B}^{\alpha}$. Also $\mathfrak{B}=\bigcup_{\gamma \in \Gamma} \mathfrak{B}^{\gamma}$. It follows at once from the corollary to Lemma 4 that $\mathfrak{B}$ can be ordered. 
Note that the theorem remains true without the restriction that $G$ is $d$-closed. For if $G$ has well ordered rank (that is, $\Gamma$ is well ordered), then so does the $d$-closure of $G$.

Corollary I. If $A$ is an abelian o-group with well ordered rank, and $\mathfrak{Y}^{*}$ is the group of all o-automorphisms of $A$ that induce the identity automorphism on each of the components of $A$, then $\mathfrak{A}^{*}$ can be ordered.

Let $\pi$ be a value preserving $o$-isomorphism of $G$ into $G$. That is, $g \in G^{\gamma} \backslash G_{\gamma}$ if and only if $g \pi \in G^{\gamma} \backslash G_{\gamma}$. Define $\left(G_{\gamma}+g\right) \pi^{\prime}=G_{\gamma}+g \pi$ for all $g \in G_{\gamma}$. Then $\pi^{\prime}$ is an $o$-isomorphism of $G^{\gamma} / G_{\gamma}$ into $G^{\gamma} / G_{\gamma} . T\left(\Gamma, D_{\gamma}\right)$ is isomorphic to the semigroup of all value-preserving $o$-isomorphisms of $G$ into $G$ that induce an $o$-automorphism on each of the components $G^{\gamma} / G_{\gamma}$ of $G$. In the proof of the theorem it was shown that $T=U$.

CoROllary II. If $\Gamma$ is well ordered, then every value preserving oisomorphism of $G$ into $G$ that induces an o-automorphism on each of the components of $G$ is an o-automorphism of $G$.

REMARK. Corresponding to Lemma 2 we have the following: Every value preserving o-isomorphism of $G$ into $G$ is an o-automorphism if and only if $\Gamma$ is well ordered and no $G^{\gamma} / G_{\gamma}$ admits an o-isomorphism onto a proper subgroup of itself. It is not necessary to assume that $G$ is $d$-closed, since both conditions imply that $G$ is $d$-closed. The proof is a consequence of Lemma 2, and the embedding theorem [1, p. 19].

If $\Gamma$ is well ordered, then by the corollary to Lemma $2, \mathfrak{A} / \mathfrak{B}$ is isomorphic to the group of all diagonal matrices in $T\left(\Gamma, D_{\gamma}\right)$. The $o$ automorphism group of the subgroup $D_{\gamma}$ of the reals is isomorphic to a subgroup of the positive reals, hence it has a natural order. Therefore $\mathfrak{A} / \mathfrak{B}$ can be ordered. Thus an ordering of $\mathfrak{B}$ can be extended to an ordering of $\mathfrak{A}$ so that $\mathfrak{B}$ is a convex subgroup if and only if $\alpha^{-1} \beta \alpha$ is positive for $\alpha \in \mathfrak{A}$ and $\beta \in \mathfrak{B}$ whenever $\beta$ is positive in $\mathfrak{B}$ (Levi [4]). In $\S 4$ we show that $\mathfrak{B}$ can be so ordered, provided that the components of $G$ admit only rational $o$-automorphisms. In $\S 3$ we order $\mathfrak{A}$ by the method we used to order $\mathfrak{B}$.

3. The group $\mathfrak{A}$. Throughout this section assume that $G$ is a d-closed abelian o-group with well ordered rank. Let $\mathfrak{A}_{\gamma}\left(\mathfrak{H}^{\gamma}\right)$ be the group of all $o$-automorphisms of $G_{\gamma}\left(G^{\gamma}\right) . G^{\gamma}=G_{\gamma} \oplus D_{\gamma}$. Thus $\mathfrak{P}^{\gamma}$ is isomorphic to the multiplicative group $\mathfrak{M}$ of all matrices of the form

$$
[a]=\left[\begin{array}{ll}
a_{11} & \theta \\
a_{21} & a_{22}
\end{array}\right]
$$

where $a_{11} \in \mathfrak{A}_{\gamma}, a_{21} \in H\left(D_{\gamma}, G_{\gamma}\right)$ and $a_{22}$ is an $o$-automorphism of $D_{\gamma}$. 
In particular, $\mathfrak{A}_{\gamma}$ is isomorphic to a subgroup of $\mathfrak{A}^{\gamma}$. Suppose that $\mathfrak{A}_{\gamma}$ and $H$ are ordered and define $[a]$ positive if $a_{22}>1$ or $a_{22}=1$ and $a_{11}>1$ or $a_{22}=1, a_{11}=1$ and $a_{21}>\theta$. Then it is easy to verify that for any nonzero matrix $a$ in $\mathfrak{M}$, either $a$ is positive or $a^{-1}$ is positive. Also if $a$ and $b$ are positive matrices in $\mathfrak{M}$, then $a b$ is positive. Finally suppose that $a, b \in \mathfrak{M}$ and $b$ is positive.

$$
a^{-1} b a=\left[\begin{array}{ll}
a_{11}^{-1} b_{11} a_{11} & \theta \\
a_{22}^{-1}\left(b_{21} a_{11}+b_{22} a_{21}-a_{21} a_{11}^{-1} b_{11} a_{11}\right) & b_{22}
\end{array}\right] .
$$

If $b_{22}>1$ or $b_{22}=1$ and $b_{11}>1$, then $a^{-1} b a$ is positive. Suppose that $b_{22}=1, b_{11}=1$ and $b_{21}>\theta$. Then

$$
a^{-1} b a=\left[\begin{array}{ll}
1 & \theta \\
a_{22}^{-1} b_{21} a_{11} & 1
\end{array}\right] .
$$

Thus if we wish to extend the order of $\mathfrak{A}_{\gamma}$ to an order of $\mathfrak{A}^{\gamma}$ we must order $H\left(D_{\gamma}, G_{\gamma}\right)$ so that $a_{22}^{-1} b_{21} a_{11}$ is positive. Now without loss of generality, $D_{\gamma}$ is a $d$-closed subgroup of the reals. Hence the only $o$-automorphisms of $D$ are multiplications by some positive real numbers. In particular, a multiplication by a positive rational is an $o$-automorphism, since $D$ is $d$-closed.

LEMMA 5. If the only o-automorphisms of $D_{\gamma}$ are multiplications by positive rationals, then $H\left(D_{\gamma}, G_{\gamma}\right)$ can be ordered so that if $h \in H$ is positive, then $\alpha h \beta$ is positive for all o-automorphisms $\alpha$ and $\beta$ of $D_{\gamma}$ and $G_{\gamma}$ respectively.

Proof. Choose and well order a basis $d_{1}, d_{2}, \cdots$ for $D$. Define $h \in H$ positive if $h \neq \theta$ and $d_{i} h>0$, where $d_{i}$ is the first basis element for which $d_{i} h \neq 0 . d_{j} \alpha h \beta=\left(r d_{j}\right) h \beta=\left(r\left(d_{j} h\right)\right) \beta$, where $r$ is a positive rational and $\alpha$ and $\beta$ are $o$-automorphisms of $D_{\gamma}$ and $G_{\gamma}$. Thus $d_{j} \alpha h \beta$ is positive if and only if $d_{j} h$ is positive.

THEOREM 2. If $G$ is an abelian d-closed o-group with well ordered rank and each component $G^{\gamma} / G_{\gamma}$ has its group of o-automorphisms isomorphic to the positive rationals, then $\mathfrak{A}$ can be ordered so that $\mathfrak{B}$ is a convex subgroup.

The proof is entirely similar to the proof of Theorem 1.

Corollary. Any torsion free abelian group can be ordered in such a way that the resulting group of o-automorphisms can also be ordered.

Proof. Let $A$ be a torsion free abelian group. Choose and well order a basis $a_{1}, a_{2}, \cdots$ for the $d$-closure $A^{*}$ of $A$. Then $A^{*}$ 
$=\Re a_{1} \oplus \Re a_{2} \oplus \cdots$. Define $a=\cdots+r_{i} a_{i}+\cdots$ positive if $a \neq 0$ and the nonzero coefficient $r_{i}$ with greatest subscript is positive. Then $A^{*}$ satisfies the hypotheses of the theorem. Hence the group of $o$ automorphisms $\mathfrak{A}^{*}$ of $A^{*}$ can be ordered, and the group $\mathfrak{A}$ of $\boldsymbol{o}$-automorphisms of $A$ is isomorphic to a subgroup of $\mathfrak{A}^{*}$.

REMARK. Note that $\mathfrak{Q}^{*}$ is isomorphic to the group $K$ of all row finite triangular matrices $\left[\pi_{\alpha \beta}\right]$, where $\alpha, \beta \in \Gamma$ the rank of $A^{*}, \pi_{\alpha \alpha}$ is a positive rational, $\pi_{\alpha \beta}$ is a rational, and $\pi_{\alpha \beta}=0$ for $\alpha<\beta$. Then $K$ can be ordered. In fact, the following definition orders $K$. $\left[\pi_{\alpha \beta}\right]$ is positive if (a) there exists an $\alpha \in \Gamma$ such that $\pi_{\alpha \alpha}>1$ and $\pi_{\beta \beta}=1$ for all $\beta<\alpha$, or (b) $\pi_{\alpha \alpha}=1$ for all $\alpha \in \Gamma$ and there exist $\alpha, \beta \in \Gamma$ such that $\pi_{\alpha \beta}>\theta$, all elements above $\pi_{\alpha \beta}$ in the diagonal containing $\pi_{\alpha \beta}$ and parallel to the main diagonal are zero, and all elements in the diagonals parallel to but distinct from the main diagonal and above the diagonal containing $\pi_{\alpha \beta}$ are zero.

4. A generalization of Theorem 2. Our proof of Theorem 2 does not permit the dropping of the hypothesis that $G$ is $d$-closed. For example, if one of the components of $G$ is isomorphic to $\Im \oplus \Im 2^{1 / 2}$, where $\Im$ is the group of integers, then the corresponding components of the $d$-closure of $G$ is isomorphic to $\Re \oplus \Re 2^{1 / 2}$. The $o$-automorphism group of $\Re \oplus \Re 2^{1 / 2}$ is isomorphic to the group of all positive elements of $\Re \oplus \Re 2^{1 / 2}$.

THEOREM 3. If $G$ is an abelian o-group with well ordered rank, and each component $G^{\gamma} / G_{\gamma}$ has its group of o-automorphisms isomorphic to a subgroup of the positive rationals, then $\mathfrak{A}$ can be ordered.

Proof. Well order -3 the elements of $G$ so that if $a, b \in G$ and $V(a)<V(b)$, then $a-3 b$, where $V(a)$ is the value of $a$ with respect to the given order of $G$. That is,

$$
\frac{0-3 g_{11}-3 g_{12}-3 \cdots g_{21}-3 g_{22}-3 \cdots}{G^{1} \backslash G_{1}} \cdot \cdots \frac{g_{\omega 1}-3 g_{\omega 2}-3 \cdots G_{2}}{G^{2} \backslash G^{\omega} \backslash G_{\omega}} \cdots
$$

For each $\pi \neq 1$ in $\mathfrak{A}$ there exists a least element $g$ in the well ordering -3 such that $g \pi \neq g$. Denote this least element by $L(\pi)$, and define $\pi$ positive if $L(\pi) \pi>L(\pi)$. If $\pi \neq 1$ and $\pi$ is not positive, then $L\left(\pi^{-1}\right)$ $=L(\pi)$ and $L(\pi) \pi<L(\pi)$. Thus $L\left(\pi^{-1}\right)=L(\pi)<L(\pi) \pi^{-1}=L\left(\pi^{-1}\right) \pi^{-1}$, hence $\pi^{-1}$ is positive. Let $\alpha$ and $\beta$ be positive elements of $\mathfrak{A}$. If $L(\alpha)=L(\beta)$ or $L(\alpha)-3 L(\beta)$ and $g-3 L(\alpha)$, then $g \alpha \beta=g \beta=g$ and $L(\alpha) \alpha \beta>L(\alpha) \beta \geqq L(\alpha)$. If $L(\beta)-3 L(\alpha)$ and $g-3 L(\beta)$, then $g \alpha \beta=g \beta=g$ and $L(\beta) \alpha \beta=L(\beta) \beta>L(\beta)$. Therefore $\alpha \beta$ is positive.

Finally suppose that $\alpha, \beta \in \mathscr{U}$ and that $\beta$ is positive. Let $g \in G^{\gamma} \backslash G_{\gamma}$. 
Then $g \alpha \equiv(m / n) g \bmod G_{\gamma}$, hence $n(g \alpha)=m g+d$ where $m$ and $n$ are positive integers and $d \in G_{\gamma}$. In particular, $d-3 g$. If $g-3 L(\beta)$, then $n\left(g \alpha \beta \alpha^{-1}\right)=(m g+d) \beta \alpha^{-1}=(m g+d) \alpha^{-1}=n g$, hence $g \alpha \beta \alpha^{-1}=g$. If $g$ $=L(\beta)$, then $n\left(L(\beta) \alpha \beta \alpha^{-1}\right)=(m L(\beta)+d) \beta \alpha^{-1}=(m L(\beta) \beta+d) \alpha^{-1}$ $>(m L(\beta)+d) \alpha^{-1}=n L(\beta)$, hence $L(\beta) \alpha \beta \alpha^{-1}>L(\beta)$. Therefore $\alpha \beta \alpha^{-1}$ is positive and $\mathfrak{A}$ is ordered.

Note that for this ordering of $\mathfrak{A}, \mathfrak{B}$ is not a convex subgroup. However, the above definition does order $\mathfrak{B}$ for any abelian $\boldsymbol{o}$-group with well ordered rank. Thus we have a second proof of Theorem 1. Moreover, this ordering of $\mathfrak{B}$ can be extended to an ordering of $\mathfrak{A}$ provided that each component of $G$ has its group of $o$-automorphisms isomorphic to a subgroup of the positive rationals. For if $\alpha \in \mathfrak{A}, \beta \in \mathfrak{B}$ and $\beta$ is positive, then the above proof shows that $\alpha \beta \alpha^{-1}$ is positive. Thus we have a proof of Theorem 2 that does not use the hypothesis that $G$ is $d$-closed.

\section{BIBLIOGRAPHY}

1. P. Conrad, Embedding theorems for abelian groups with valuations, Amer. J Math. vol. 75 (1953) pp. 1-29.

2. - Extensions of ordered groups, Proc. Amer. Math. Soc. vol. 6 (1955) pp. 516-528.

3. A. G. Kurosh, The theory of groups, Chelsea, New York.

4. F. W. Levi, Ordered groups, Proceedings of the Indian Academy of Sciences, vol. 16 (1942) pp. 256-263.

TUlane UNIVERSity 